\title{
„Blasse Mädchen“ im Spiegel - Zur Berichterstattung über Politi- kerinnen im Wahlkampfjahr 2013 am Beispiel von Medientexten über Katja Kipping
}

\author{
Anke Heier (Kopenhagen)
}

\begin{abstract}
This paper examines how the news magazine Der Spiegel and the online platform Spiegel Online $(S P O N)$ portrayed the politician Katja Kipping from the German party Die LINKE during the election campaign in 2013. Kipping is since 2012 chairman of the party Die LIN$\mathrm{KE}$ and contributes to the increasing number of women in politics in recent years. The debate on sexism in everyday life and politics and the discussion related to the appointment of Ursula von der Leyen as the first female German defense minister has shown that an increasing number of women in politics does not automatically lead to the opinion that women are suitable for a political office. Since der Spiegel is one of Germany's most influential weekly news magazines, it is important to be aware of the contribution it makes to a person's image in the media and the influence on the election campaign, because if this image is undifferentiated, the reader may reduce the image even more. This reinforces the process of stereotyping. The purpose is to analyze to what extent der Spiegel and SPON spread a unilateral or a differentiated picture or concept and what features of the investigated person that are highlighted. Methodologically, the study is based on the questionnaire for frame analysis by Ziem, modifies it and applies a sequence of questions adapted to female politicians.
\end{abstract}

\section{$1 \quad$ Motivation und Fragestellung}

Die öffentlich-politische Diskussion des Jahres 2013 in Deutschland war zu einem großen Teil geprägt von Wahlkämpfen und Koalitionsverhandlungen, nicht zuletzt auf der Bundesebene. Sowohl Politik als auch Medien trugen dazu bei, nicht nur Themen und Ziele der Parteien vorzustellen und zu diskutieren, sondern auch die Fähigkeiten und Handlungen der zu wählenden bzw. gewählten PolitikerInnen zu beurteilen. Als damals amtierende und potenzielle zukünftige Kanzlerin zog Angela Merkel herausragende Aufmerksamkeit auf sich. Aber auch andere Frauen wie Manuela Schwesig, Ursula von der Leyen, Katja Kipping und Katrin Göring-Eckardt wurden aufgrund ihrer hohen politischen Ämter bzw. ihres Status als Anwärterinnen auf Ministerposten zum Thema von Pressetexten und Personaldiskussionen. Zusammen mit 36,1 \% oder 228 anderen weiblichen Abgeordneten (cf. DDB s. a., Stand Dezember 2014) - der höchste Anteil von Politikerinnen im Bundestag seit 1949 - beweisen sie die immer größer und normaler werdende Präsenz von Frauen in der deutschen Politik. Andererseits

Linguistik online 79, 5/2016 - http://dx.doi.org/10.13092/lo.79.3332

CC by 3.0 
kam es am Anfang des Jahres 2013 zu einer Debatte über Sexismus im Alltag, in den Medien und in der Politik, ausgelöst durch das Verhalten des Spitzenkandidaten Rainer Brüderle (FDP) gegenüber der Journalistin Laura Himmelreich. Und am Ende des Jahres wurde die als überraschend empfundene Ernennung von der Leyens zur ersten Verteidigungsministerin diskutiert. Gerade das letzte Ereignis verweist darauf, dass die erhöhte Präsenz von Frauen in der Politik nicht automatisch die Auffassung nach sich ziehen muss, selbstverständlich seien Frauen für ein politisches Amt geeignet bzw. grundsätzlich (politisch) kompetent. ${ }^{1}$

Dies sind Gründe, sich mit der öffentlichen, genauer print- und onlinemedialen Thematisierung von Politikerinnen eingehender zu beschäftigen. Am Beispiel der Person Katja Kipping, mit Fokus auf die Berichterstattung über sie im genannten Jahr 2013 und einem vergleichenden Blick auf 2015 sowie mit Beschränkung auf die Quellen Spiegel und Spiegel Online $(S P O N)$ soll in diesem Beitrag gefragt werden, welches Bild von einer (wahlkämpfenden) Politikerin gezeichnet wird und welche sprachlichen Mittel dafür zum Einsatz kommen. Lässt es sich als differenziert charakterisieren oder neigt es dazu, bestimmte (angenommene) Merkmale immer wieder hervorzuheben und so zu einer Stereotypisierung der Politikerin beizutragen. Ein besonderes Interesse liegt dabei auf der Kommentierung der sprachlichen Performance der Person. Damit will der Aufsatz die bisherigen Erkenntnisse zum Frauenbild in Deutschland, spezieller zu dem von Politikerinnen in den deutschen Medien, ergänzen. Er wird ebenfalls dazu genutzt, den zur Beantwortung dieser Fragen zusammengestellten Fragenkatalog, mit dessen Hilfe die Texte analysiert werden und der seinen Ausgangspunkt in der linguistischen Frame-Semantik nimmt, auszuprobieren und zur Diskussion zu stellen, denn er enthält einige, m. E. relevante Anpassungen an die hier interessierende Personenbzw. Berufsgruppe, wodurch die obigen Fragen genauer beantwortet werden sollen.

\section{Quellenbasis und Personenauswahl}

Anstatt vereinzelte Porträts aus verschiedenen Jahren sowie Zeitungen und Magazinen auszuwählen und zu betrachten, habe ich mich dafür entschieden, sämtliche schriftliche Berichterstattung jeweils eines ganzen Jahres (2013 und zum Vergleich 2015) zu erfassen. Denn ich gehe davon aus, das nicht nur Personenporträts an Imagebildung bzw. -verfestigung beteiligt sind, sondern auch - und nicht wenig - die stete Wiederholung bestimmter Beschreibungen und Wertungen in Meldungen und Nachrichten. Eine Beschränkung fand in Bezug auf das untersuchte Presseorgan sowie auf die thematisierten Personen statt. Diese Beschränkung führt dazu, dass diese Studie zwar keine generalisierbaren Ergebnisse zur medialen Auffassung über deutsche Politikerinnen liefert, aber ein Spiegel -Bild erfasst, das seinen Beitrag zur Entstehung bzw. Festigung eines umfassenderen Bildes der untersuchten Person und letztlich doch von Politikerinnen überhaupt leistet.

\footnotetext{
${ }^{1}$ Cf. Holtz-Bachas Überblick (2007b: 7-16) zur Entwicklung der Berichterstattung über Frauen und besonders Politikerinnen seit der 1970er Jahren in Deutschland, dessen Fazit ist, dass trotz erhöhter politischer Beteiligung von Frauen ihre Leistungen und damit ihre Fähigkeiten noch in der 2000er Jahren abgewertet werden. Meyer (2009) verweist auf den noch immer wirkenden Zusammenhang zwischen Geschlechterrollenstereotypen und Kompetenzauffassungen gegenüber Politikerinnen, z. B. in Bezug auf eine Ressortverteilung, cf. dazu auch Holtz-Bacha (2007a: 82).
} 
Das Nachrichtenmagazin Der Spiegel als eine Quellenbasis zu nutzen gründet sich in seinem Status, ein Leitmedium in der deutschen Presselandschaft zu sein, wodurch es meinungsbildend über seine Leserschaft hinaus wirkt, und zwar auch in Bezug auf das Image von Personen. Die Wirkung des Blattes mit einer Auflagenzahl von knapp 875.000 und Reichweite von 5,87 Mio. Lesern im Jahr 2013 (cf. STATISTA s. a.) wird also durch Zitate in anderen Medien noch verstärkt. Das wöchentliche Erscheinen sollte eine ausführlichere Präsentation von Themen ermöglichen als beispielsweise das kontinuierlich und tagesaktuell publizierende Online-Angebot des Verlages, weshalb auch eine ausführlichere Besprechung von Personen erwartet wird. Die Plattform SPON gilt in der deutschen Online-Nachrichtenwelt ebenfalls als vorbildhaft. Sie hatte 2014 rund 204 Mio. Besucher, verbreitet aber eben eher Meldungen als Reportagen (cf. STATISTA s. a.). Zu einem geringen Teil erscheint im Spiegel wie im SPON dasselbe. ${ }^{2}$ Beide Organe ergänzen sich so in ihrer Form der Berichterstattung, aber auch in ihrem Leserkreis. Die redaktionellen Leitlinien des Blattes - das politische Profil - beschreiben Maurer/Reinemann (2006: 130) als linksliberal. Dies lässt vermuten, dass linke und linksliberale PolitikerInnen mit eher wohlwollenden, vielleicht auch differenzierteren Besprechungen rechnen können als konservative Personen, was bei einer Interpretation der Daten zu berücksichtigen ist.

Das mediale Image Katja Kippings zum Gegenstand der Untersuchung zu machen rührt daher, dass Kipping spätestens seit 2012 als Vorsitzende der Partei Die LINKE zu den Spitzenpolitikerinnen Deutschlands gehört, wenn auch als Mitglied einer Oppositionspartei. Aber einmal nicht Regierungsmitglieder oder PräsidentschaftskandidatInnen zum Thema zu machen, könnte zu interessanten Einsichten führen. Die LINKE ist durch ihre besondere Entstehungsgeschichte zudem eine umstrittene Oppositionspartei und Kipping eine Ostdeutsche und auf einem Spitzenposten junge Politikerin, was m. E. medialen Gesprächsstoff bieten sollte. Andererseits hat auch die überschaubare Quellenlage zu Kipping (siehe mehr dazu unter Punkt 3.2) dazu geführt, gerade an der Berichtserstattung über sie den Fragenkatalog zu erarbeiten und auszuprobieren.

\section{Theoretische und methodische Überlegungen}

\subsection{Zum Begriff des Stereotyps}

Die Frage nach dem Spiegel-Bild von (wahlkämpfenden) Politikerinnen und dessen möglicher Stereotypie bzw. dessen Beitrag zur Stereotypenbildung fordert die Klärung des Begriffes Stereotyp, wie er in diesem Beitrag verwendet wird. Die bisher benutzten Begriffe Bild und Image verweisen auf die Lippmann'sche Beschreibung von Stereotypen als ,pictures in our heads“ (Lippmann 1922: 125), verstanden als kognitive Schematisierungen, die unsere Wahrnehmung vorstrukturieren. Sehr oft werden sie mit gruppenbezogenen Selbst- und Fremdbildern in Verbindung gebracht, deren Exemplare die Merkmalszuschreibungen der Gruppe übernehmen. In unserem Fall haben wir es mit der Gruppe der Frauen ${ }^{3}$, der der PolitikerInnen und der von Parteien zu tun. Eine weitere Auslegung - wie z. B. bei Konerding und Quast-

\footnotetext{
2 Eigene Beobachtung aus dem Vergleich der gefundenen Artikel heraus.

${ }^{3}$ Das Geschlecht als Gruppenmerkmal zu verwenden ist umstritten. In der Soziolinguistik wird eher von einer Superkategorie gesprochen (cf. Veith 2005: 152).
} 
hoff -, die auch bereits bei Lippmann angelegt ist, schließt Einzelpersonen als stereotypisierte Objekte ebenso ein wie Gegenstände, Ereignisse und Sachverhalte im Allgemeinen. So schreibt Quasthoff bereits 1973 und wieder 1998: „Ein Stereotyp ist der verbale Ausdruck einer auf soziale Gruppen oder einzelne Personen als deren Mitglieder gerichteten Überzeugung, die in einer gegebenen Gemeinschaft weit verbreitet ist. Es hat die logische Form eines Urteils, das in ungerechtfertigt vereinfachender und generalisierender Weise, mit emotional wertender Tendenz, einer Klasse von Personen bestimmte Eigenschaften oder Verhaltensweisen zu- oder abspricht" (Quasthoff 1998: 48). Weil diese Untersuchung auf Einzelpersonen fokussiert, soll hier in Anlehnung an Konerding (2001: 152f.) auch von konsensuellem koorientierendem, schematisiertem Alltagswissen über (etwas bzw.) jemanden gesprochen werden, das insofern handlungsleitend wirkt, als es Interpretationen und Wertungen vorgibt, welche nicht zuletzt durch ihre sprachliche Formulierung transportiert bzw. tradiert werden. ${ }^{4}$ Handlungsleitend bedeutet im Rahmen von Wahlkämpfen unter anderem, dass die einzelnen Bürger aufgrund ihres Bildes von kandidierenden PolitikerInnen, welches sie auch durch die Medienberichterstattung aufbauen oder ihr entnehmen, eine Wahlentscheidung treffen. Daneben soll die komplexitätsreduzierende Wirkung von Stereotypen hervorgehoben werden, durch die die Vielschichtigkeit von Individuen und ihr situationsbegründetes Handeln in der Regel nicht gut wahrgenommen werden. Fällt ein mediales Bild also schon verkürzt aus, kann es beim Leser zu noch stärkerer Reduktion kommen, was den Prozess der Stereotypisierung, verstanden als Etablierung konsensueller Wahrnehmungs- und Evaluationsschemata, in eine Richtung hin zu nicht nur festen, sondern auch falschen Vorstellungen führt.

Auf ihrer sprachlichen Seite, die hier im Fokus der Betrachtung steht, entsprechen Stereotype formelhaften Ausdrucksweisen wie Redewendungen und Sprichwörtern, stabilen lexikalischen Verbindungen wie Wortbildungen und Kollokationen sowie wiederholt gebrauchten freien Wortgruppen und Sätzen, in denen die erwähnten Gegenstände, Personen bzw. Sachverhalte eine Prädikation erfahren. Ich gehe davon aus, dass v. a. die letzte Gruppe in der Untersuchung relevant wird, weshalb nicht nur auf Redewendungen und Kollokationen zu achten ist.

\subsection{Zur Korpuserstellung, Datenererhebung und Datenaufbereitung}

Als Untersuchungsbasis dienen 45 Artikel aus allen Ausgaben des Nachrichtenmagazins Der Spiegel aus dem Jahr 2013 sowie aus SPON. Der Datenbank Nexis Lexis Academic, die alle Artikel des Print- und Online-Mediums enthält, wurden diejenigen Artikel entnommen, die die Suchbegriffe Kipping und Kippings enthielten. Doppelungen durch Mehrfachpublikation wurden ausgeschieden, ebenso Artikel, die sich nicht auf die Berichterstattung über die betreffende Person beziehen, mit Kipping also etwas bzw. jemand anderes gemeint ist. So blieben 45 Artikel übrig. Für 2015 wurden in derselben Weise 33 Artikel ermittelt. Diese Zahlen sind für eine Berichterstattung über eine Bundespolitikerin mit Spitzenposition nicht groß. Im Vergleich dazu erhielten Manuela Schwesig (SPD) und Katrin Göring-Eckardt (Bündnis 90/die Grünen) - beide Spitzenpolitikerinnen ihrer ebenfalls linken Parteien und Kandidatinnen für Ministerposten in der Bundestagswahl in 53 bzw. 132 Artikeln mediale Aufmerksam-

\footnotetext{
4 Ähnlich zusammengefasst habe ich den Stereotypenbegriff auch in Heier (2016: 16).
} 
keit. Auch über Gregor Gysi - wie Kipping Bundespolitiker und MdB, jedoch Fraktionsvorsitzender der LINKEN und vom Spiegel als Spitzenkandidat bei den Wahl 2013 gehandelt wird in 146 Artikeln, also deutlich mehr berichtet.

In einem weiteren Schritt wurden diejenigen Sätze und Phrasen zusammengetragen, die in Relation zur betreffenden Person stehen. In der Regel handelt es sich um die unmittelbare textuelle Umgebung, in der der Name auftritt. Darüber hinaus wurden alle weiteren Aussagen zur Person im jeweiligen Text berücksichtigt, wobei auf den mit der Person verbundenen anaphorischen bzw. kataphorischen Gebrauch von Pronomen sowie auf Titel bzw. Funktionen zu achten und ihre Textumgebung zu berücksichtigen war. Es wurden also alle Texte komplett gelesen und nicht nur automatisch die sprachliche Umgebung der Namen erfasst, was bei 45 Artikeln noch möglich war.

Im nächsten Schritt diente ein Fragenkatalog der Ordnung der gefundenen sprachlichen Einheiten. Dieser Katalog wurde in Auseinandersetzung mit verschiedenen früheren Systematisierungsversuchen von personen- und gruppenbezogenen Aussagen (z. B. von van Dijk 1984; Klein 1998; Ziem 2008a, 2008b) und mit Blick auf die Hauptfragen des Aufsatzes erstellt. Genauer gesagt bilden frameanalytische Ansätze zur Erfassung der Bedeutung von Konzepten seine Basis. Unter Frame wird hier einerseits ein Wissensrahmen, eine kognitive Einheit verstanden, in der sich aufgrund ihrer eigenen Strukturiertheit Wissen zu einem Konzept organisiert. Dieses Wissen gilt als verfestigt und konventionalisiert, wird aber in konkreten Situationen kontextualisiert und konkretisiert (cf. Busse 2012: 530). Darauf aufbauend dienen Frames, genauer Matrixframes, wie sie insbesondere von Konerding (1993) herausgearbeitet und von Fraas (1996), Klein (2002) und Ziem (2008a, 2008b) für die Diskursanalyse bearbeitet worden sind, als Analyseinstrument ${ }^{5}$ für die Bedeutung eines Ausdrucks, indem die Slots oder Inhaltskategorien des Matrixframes, dem der zu untersuchende Begriff hyponym untergeordnet ist und dadurch seine Slots, formulierbar als sinnvolle Fragen, erbt, an einen Text bzw. ein Textkorpus herangetragen werden. Die Äußerungen in den Texten stellen die konkreten Füllwerte (fillers) dar. Ihre Wiederholung im Diskurs ist nach Ziem (2008a: 106) ein Zeichen für ihr Potenzial, zu Standardwerten (default values), d. h. zu nicht mehr zu thematisierenden, kollektiv geteilten, schematisierten Wissenselementen zu werden, was dem oben skizzierten Stereotypenbegriff sehr nahekommt. Nach Klein (1998: 38-42) bietet sich das Format des Frames darum auch gut für Stereotypenanalysen an. In diesem konkreten Zusammenhang kann gefragt werden, welchen Beitrag der Spiegel zur Etablierung von Standardwerten leistet bzw. ob überhaupt ein Frame gefüllt ist, wenn ja womit und wie differenziert und ob bestimmte Slots mehr und ähnlicher bedient werden als andere.

Wie wurde der Fragenkatalog erstellt? Nachdem van Dijks (1984: 37) Kategorien „Aussehen“, „ökonomischer Status“, „Normen/Glauben/Sprache“, „Verhalten“ und „Charakter/Eigenschaften“ zur Erfassung von stereotypen Aussagen ausprobiert und als für eine Personencharakterisierung zu grob verworfen worden sind, lehnt sich der Analysekatalog nun an den auf Konerding basierten Fragenkomplex von Ziem (2008b: 416-418) zum Matrixframe Personen in berufsbezogener Rolle an, ohne auch ihm völlig zu folgen. Ich gehe nicht derart ins Detail wie Ziem bzw. Konerding, sondern orientiere mich an den übergeordneten Prädika-

\footnotetext{
${ }^{5}$ Cf. dazu auch den kurzen, prägnanten Überblick in Niehr (2014: 76-80).
} 
torenklassen, versuche darüber hinaus bestimmte Fragen zu bündeln ${ }^{6}$ und an den hier relevanten Beruf anzupassen. Dabei erscheint mir vor dem Hintergrund der gefundenen Texte sowie früherer medienwissenschaftlicher Untersuchungsergebnisse zum Thema PolitikerInnen in den Medien (cf. z. B. Drinkmann/Caballero 2007)7 eine stärkere Berücksichtigung von Fragen über Privates relevant, da PolitikerInnen nicht nur in ihrem beruflichen Umfeld präsentiert werden. ${ }^{8}$ M. E. gehören die private Seite bzw. Persönlichkeitsmerkmale einer Person in dem Moment, wo es nicht um eine Berufsgruppe, sondern um Einzelpersonen geht, zum Frame dieser Person hinzu. Eine Ausklammerung der privaten Seite zugunsten der beruflichen bzw. eine starke Trennung der beiden Bereiche scheint mir außerdem eine tendenziell kulturspezifische Sichtweise auf Personen zu sein. Eine rein induktive Gewinnung von thematischen Kategorien aus dem Untersuchungsmaterial - also ohne Bezug auf Matrixframes - wäre auch möglich und könnte zumindest zeigen, welche Slots in den Texten eine explizite Rolle spielen. Jedoch lassen sich dadurch eventuelle Lücken in der Beschreibung der Begriffe, hier Personen, nicht gut erkennen. Da es mir daneben um die Erfassung der verwendeten sprachlichen Mittel geht, die die Journalisten für die Beschreibung des kommunikativen Verhaltens der Politikerin verwenden, wurde der Fragenkatalog außerdem in diese Richtung erweitert. Insbesondere Sprachhandlungsverben und mit ihnen verbundene Adjektive und Adverbien zeigen Interpretationen und Beurteilungen durch die Journalisten an. Diese können einerseits als Kommentare zu einer Fähigkeit, die PolitikerInnen besitzen sollen - wie verbale Überzeugungs- und Durchsetzungskraft, Vermittlungs- und Integrationsvermögen ${ }^{9}$ - gedeutet werden, andererseits als Bewertung ihrer politischen Aktivitäten. Dass politisches Handeln im hohen Maße sprachliches Handeln ist, ist ein Grundgedanke in der Politolinguistik (cf. Girnth 2002: 1-3; Niehr 2014: 11f.; Schröter/Carius 2009: 9).

Es folgt der zugrunde gelegte Fragenkatalog. Die Sätze wurden so formuliert, dass sie nach Zuschreibungen fragen, nicht nach ontisch deutbaren Merkmalen.

1. Name: Wer ist $\mathrm{x}$ ? Wie wird die Person bezeichnet? (Name, Titel, Spitzname, Rolle/Funktion, Mitgliedschaft, Position in der Partei oder einer anderen Gruppe)

2. Eigenschaften: Wie ist $x$ ? Welche äußeren und inneren Merkmale und Fähigkeiten werden der Person zugeschrieben? (allgemeine Charakteristika) Werden allgemeine sprachliche bzw. kommunikative Merkmale thematisiert?

3. Privater Hintergrund: Wie entwickelt sich x privat? Mit welchem vergangenen, gegenwärtigen und zukünftigen privaten Werdegang wird die Person verbunden?

\footnotetext{
${ }^{6}$ Ein ähnliches Vorgehen in Bezug auf die Bündelung der Matrixframe-Slots stellt Wengeler (2012: 61f.) für den Frame „Ereignis“ vor.

${ }^{7}$ Drinkmann/Caballero (2007) haben in der Berichterstattung sowohl über PolitikerInnen bzw. PräsidentschaftskandidatInnen private Themen als bedeutenden Faktor herausgearbeitet.

${ }^{8}$ Die Beschreibung der privaten Seite von Personen ließe sich auch als ein Frame neben dem Frame Person in berufsbezogener Rolle auffassen. So differenzieren Drinkmann/Caballero (2007) in Privatleben, Persönlichkeit, Politik und Gender, wenn sie die Berichterstattung über die PräsidentschaftskandidatInnen Köhler und Schwan 2004 auswerten. Letztlich bleibt aber die übergeordnete Kategorie Person bestehen.

${ }^{9}$ Siehe dazu z. B. die Angaben zu Funktionen politischer Kommunikation in Girnth (2002: 38-43): regulativ, poskativ, informativ, persuasiv, integrativ.
} 
4. Beruflicher und ideologischer Hintergrund: Wie entwickelt sich $\mathrm{x}$ beruflich? Mit welchem vergangenen, gegenwärtigen und zukünftigen Werdegang wird die Person verknüpft? Welche Ziele, welche politische Haltung und welche Leistungen werden $\mathrm{x}$ zugeschrieben?

5. Themen: Womit beschäftigt sich $\mathrm{x}$ ? Mit welchen Themen und Ereignissen wird die Person in Verbindung gebracht? Welche sind nichtpolitischer, welche politischer Natur? (politischer, nichtpolitischer Kontext)

6. Aktivitäten: Was und wie tut es $x$ ? Welche Handlungen werden ihr zugewiesen? Wie werden diese charakterisiert? (Handlungen, Aufgaben/Pflichten, Mittel, Mitspieler/Gegner) Wie wird ihr Kommunikationsverhalten beschrieben? (Sprachhandlungen und ihre Beurteilung)

Für die Beantwortung der Fragen wird dann einerseits auf den Inhalt, andererseits auf die konkrete Formulierung geachtet. In die Beantwortung der Fragen fließen neben quantitativen Aspekten auch Interpretationen zum Gefundenen ein.

\section{$4 \quad$ Katja Kipping in Spiegel und SPON 2013 - Analyse ${ }^{10}$}

Die 45 Artikel aus Spiegel und SPON 2013, in denen Katja Kipping namentlich erwähnt wird und die damit das zugrundegelegte Korpus bilden, inkludieren genau einen Artikel, der die Politikerin direkt zum Thema macht. Im Spiegel vom 06.01.2013 wird Kipping im Rahmen der Niedersachsenwahl im Vergleich zu ihrer Parteigenossin Sahra Wagenknecht vorgestellt. Es ist auch der einzige Bericht, in dem ihr Name - und zwar der Vorname - im Titel erscheint: Süße Katja gegen rote Sahra. Dieses Porträt lässt sich zwar in Verbindung mit dem Bundestagswahlkampf bringen, zeitlich liegt es jedoch noch recht entfernt von der eigentlichen Wahl. Die Kontrastierung deutet eher auf ein Medieninteresse an einer parteiinternen Entwicklung hin. In SPON vom 16.01.2013 erschien diesbezüglich ein Porträt über Wagenknecht, in dem diesmal Kipping als Kontrahentin Wagenknechts auftritt, am 26.08.2013. ein Porträt über Gregor Gysi, in dem der Autor ihre Aktivitäten einschätzt. Kipping selbst bleibt in diesen Artikeln aber eine Randfigur. 2015 gibt es gar kein Porträt. Die anderen gesammelten Texte, informierende Kommentare und mehr oder weniger kommentierende Meldungen ${ }^{11}$, enthalten Zitate bzw. Redewiedergaben Kippings zu verschiedenen Themen neben Beschreibungen und Interpretationen ihres Tuns. Ein richtiges Interview, in dem Kipping sich oder die Partei bzw. ihre Positionen vorstellen kann, findet sich dagegen nicht, auch nicht 2015. Eine Erklärung für diese relativ geringe personenorientierte Berichterstattung könnte darin liegen, dass Kipping nicht die alleinige Spitzenkandidatin für Wahlen, sondern Teil eines größeren Wahlkampfteams war, außerdem auch nicht den Fraktionsvorsitz im Bundestag innehat, sondern als LINKEN-Vorsitzende eher eine wichtige parteiinterne Rolle spielt. Das mediale Interesse richtete sich 2013 vielmehr auf den in der Bundespolitik etablierten, als zukünftigen

\footnotetext{
${ }^{10}$ Im Folgenden werden wörtliche Übernahmen aus den Spiegel-Texten kursiv markiert. Ich verzichte aus Platzgründen auf die Quellenangabe jedes einzelnen Belegs. Wenn nicht anders quantitativ markiert handelt es sich um einmalige Belege.

${ }^{11}$ Burger/Luginbühl (2014: 237-249) unterscheiden einerseits Kommentare und Meldungen als zwei bedeutende Pressetextsorten, verweisen zugleich auf den Trend der Vermischung der Textsorten in den heutigen Medien. Für diese Untersuchung erwies sich eine differenziertere Unterscheidung als oben vorgenommen aufgrund des Mischcharakters vieler Artikel als nicht ergiebig.
} 
Oppositionsführer gehandelten und vom Spiegel offensichtlich als medientauglicher eingeschätzten Gregor Gysi. Dieser wird interviewt, porträtiert, zitiert und privat und beruflich beobachtet, sein Kommunikationsverhalten wird als witzig, charmant und tendenziell machohaft eingeschätzt.

\subsection{Name}

In 43 von 45 Artikeln geschieht der Verweis auf die Person durch Vor- und Nachname mit und ohne den Hinweis auf ihre Funktion in der Partei, die in der Regel als Chefin (26-mal), seltener als Vorsitzende (9-mal) bezeichnet wird. Der Name der Partei variiert dabei. Sehr selten stehen nur der Nachname - etwas, was sich bei bekannteren Persönlichkeiten - auch Frauen - wie Angela Merkel oder Ursula von der Leyen häufig findet - und Bezeichnungen wie Frau Kipping oder die Linken-Frontfrau (je 1-mal). Kipping wird aber mehrmals als Teil der Parteispitze erwähnt (z. B. Co-Chefin: 5-mal) und einmal als Mitglied der Emanzipatorischen Linken, einer Gruppierung der Linkspartei, deren Zielgruppe laut Spiegel ,rotweintrinkende alphabetisierte SchalträgerInnen“ (04.02.2013) seien und nur Kipping angehöre. ${ }^{12}$ Die vielfache Wiederholung von Position und vollem Namen lässt sich dahingehend deuten, dass die Autoren die untersuchte Person als auf ihrem Posten nicht etabliert genug und für die Leser zu unbekannt beurteilen, um sie nur mit dem Nachnamen erwähnen zu können. Die Wiederholung trägt aber dazu bei, dass gerade die Verbindung von Funktion und Personenname zu einem Standard wird, durch die Wiederholung des Vornamens aber auch das Geschlecht Kippings konstante Erwähnung findet: Die Parteichefin Katja Kipping. Diese Benennung ist auch 2015 Usus, mindestens bei ihrer Ersterwähnung im Text, um an ihre Position zu erinnern. ${ }^{13}$

Im Porträt dagegen wird Katja Kipping zweideutig als rote Lady, süße Katja, das Mädchen, Jeanne d'Arc der Linken-Avantgarde, Bollwerk gegen Sahra Wagenknecht, Siegerin und schließlich als (neue) Parteivorsitzende beschrieben, wenn auch nicht alle Bezeichnungen vom Autor selbst stammen, sondern als Zitate aus den unterschiedlichen parteiinternen Lagern zu betrachten sind. Obwohl der Artikel kaum Wiederholungen und damit potenzielle Standardwerte enthält, tragen die Bezeichnungen dazu bei, ein Bild zu aufzubauen, das eine engagierte, aber nicht ganz ernstzunehmende bzw. noch nicht etablierte junge Person zeigt. Einen in den Medien eingebürgerten Spitznamen besitzt Kipping nicht, auch nicht 2015.

\subsection{Eigenschaften und Sprache}

$\mathrm{Zu}$ allgemeinen äußeren und inneren Charakteristika und Fähigkeiten Kippings finden sich in den Texten außerhalb des Porträts kaum Hinweise. Lediglich ihr Alter wird dreimal erwähnt. In einem Kommentar zu einer Polittalkshow kommt einmal ihr Geschlecht zur Sprache, da sie im Talk einen Teil der ausschließlich weiblichen Opposition bildet. Im Porträt dagegen nimmt sich der Autor Markus Deggerich den Platz, um Kippings Haare und Kleidung als farbenfroh

\footnotetext{
${ }^{12}$ Der Titel des Artikels ist im Übrigen „Kennt keine Sau“. Er bezieht sich aber nicht direkt auf Personen, sondern eher Gruppierungen innerhalb der LINKEN.

${ }^{13}$ In 32 von 33 Artikeln wird Kipping mit Vor- und Zunamen erwähnt, 13-mal als Chefin betitelt, 3-mal als eine der Chefs, 10-mal als Vorsitzende. Es gibt keinen Artikel, in dem die Zuordnung zur Partei fehlt.
} 
bzw. rot sowie optisch auffällig zu beschreiben. Letztere ist durchaus als Anspielung auf die Farbe der Partei im deutschen politischen Farbenspektrum deutbar, soll aber auch ein Hinweis auf eine innerparteiliche Sonderstellung Kippings als Vertreterin einer neuen Generation sein. ${ }^{14}$ Die Beschreibung ihres Charakters und ihrer Fähigkeiten lässt sich kaum trennen von der Einschätzung ihres politischen Verhaltens. Sie wird auf der einen Seite als süß und zunächst unscheinbar und in ihren Positionen als naiv bezeichnet - was sich auch als Beurteilung der Person deuten lässt. Andererseits werden Kipping Attribute wie modern, vielseitig, emanzipiert, machtbewusst und ohne Scheu vor Kameras zugeordnet ebenso wie im entscheidenden Moment kühl bzw. mit kühlem Machtkalkül agierend. Dann vergleicht der Autor das Auftreten Kippings mit dem Angela Merkels vor deren Wahl zur Parteivorsitzenden und Bundeskanzlerin und vermutet, dass auch Kipping unterschätzt werde.

Kippings vorherrschendes kommunikatives Verhalten wird nur wenig beschrieben. Ihr auffälliges Merkmal sei ein moderierender Ton.

Obwohl sprachlich kaum Wiederholungen in Bezug auf die oben gestellten Fragen zu finden sind, bleibt inhaltlich der Eindruck einer äußerlich auffälligen und unangepassten, charakterlich selbstbewussten, zugleich aber auch ungefestigten und unterschätzten Person, wobei das Porträt Attribute verwendet, die im größeren Maße jungen Frauen zugeordnet werden: sü $\beta$, naiv, ohne Scheu, emanzipiert. ${ }^{15}$

\subsection{Privater Hintergrund}

Kippings privater Hintergrund spielt in der Spiegel -Tagespresse und in den meisten der Magazinausgaben keine Rolle. In einem Bericht zum Straßenwahlkampf wird ein Auftritt Kippings in ihrer Heimatstadt Dresden erwähnt. Das Porträt vom 06.01.2013 zeichnet sie als Mutter einer Tochter und als Ostdeutsche bzw. Dresdnerin, die die Wende, in der Zeit ihrer Pubertät erlebt hat. 2015 ist die, wenn auch nur einmalige Erwähnung ihres Heimatortes Dresden mit Verweisen auf Pegida und das Luftbombardement von 1945 verbunden.

\subsection{Beruflicher und ideologischer Hintergrund}

Der berufliche, d. h. politische Werdegang Kippings findet nur im Porträt eine Erwähnung: Politisches Engagement an der Uni, Kontakt zum linken Jugendhaus „Roter Baum “, das in PDS-Hand lag. Andere linke Parteien seien in der Stadt kaum etabliert gewesen. Der Wunsch nach nachhaltigen politisch linken Aktivitäten führte sie zur Partei PDS. Nach einem zeitlichen Sprung in das Jahr 2012 erwähnt der Artikel, dass Kipping in einer Krise der Partei für einen Platz im Parteivorsitz kandidiert und diesen gewinnt. 2013 wird Kipping immer noch als neue Vorsitzende bezeichnet, die sich noch beweisen müsse, auch gegenüber ihrer Konkurrentin Wagenknecht und dem sogenannten Lafontaine- bzw. Fundi-Flügel. Darüber hinaus gibt es keine Karrierebeschreibungen oder Zukunftsprognosen. Wie am privaten Werdegang haben die anderen Artikel auch an der beruflichen Entwicklung der untersuchten Person kein

\footnotetext{
${ }^{14}$ In einem Artikel vom 01.10.2013 wird Kipping im Vergleich zu ihren Parteigenossen auch als zu jung, um DDR-Nostalgie aufkommen zu lassen, beschrieben. Im Porträt wird sie alten Männern gegenübergestellt.

15 Es findet sich nichts aus 2015, was in diese Kategorie eingeordnet werden könnte.
} 
Interesse. Sie fokussieren offensichtlich auf die jeweils gegenwärtigen Handlungen bzw. Aussagen der Person, ohne Kipping dabei näher vorzustellen. Ein Grund kann darin liegen, dass sie als Parteivorsitzende v. a. als Sprecherin ihrer Partei betrachtet wird. Dies bestätigt auch die Analyse der Artikel aus 2015. Hier wird lediglich zusätzlich eine besondere Nähe Kippings zur Blockupy-Bewegung erwähnt und diskutiert.

Kippings übergeordnete politische Ziele sind dagegen Themen, die nicht nur im Porträt vorkommen, sondern auch Platz in den allgemeinen Meldungen über ihr politisches Tun einnehmen. Diese sollten im Rahmen einer Berichterstattung über Politik in Bundestagswahlkampfzeiten ja auch eine herausgehobene Rolle spielen. Ein wichtiges Ziel Kippings sei es, ein Zentrum in ihrer Partei zu schaffen und die Flügelkämpfe zu überwinden, so dass in den Laden Ruhe einkehre. Ein anderes Ziel kann als Neupositionierung ihrer Partei in der Parteienlandschaft und in Bezug auf mögliche Regierungskoalitionen bezeichnet werden, was sie auch 2015 noch beschäftigt. Dazu gehört die Etablierung eines entspannten Verhältnisses zur SPD (2-mal) und die Abgrenzung zur Partei Alternative für Deutschland (AfD) genauso wie die Einführung eines Mindestlohns und - direkt nach der Bundestagswahl - die Überwindung der Konzentration des Parteiprogramms auf die Kritik an der Agenda 2010. Dies sind v. a. innerparteiliche Ziele, die für die Bürger bei ihrer Wahlentscheidung wohl kaum eine größere Rolle gespielt haben dürften.

Kippings politische Grundhaltung wird einerseits als rot, emanzipatorisch, links und zum Realo-Lager gehörend bezeichnet sowie als Bonvivant-Sozialismus beschrieben, der ein bedingungsloses Grundeinkommen ebenso beinhalte wie Lesetage, was mit den Wunsch nach Rotwein für alle verglichen wird. ${ }^{16}$ Diese Position schätzt das Porträt als geschmeidig ein, also nicht sehr festgelegt. Als Reaktion auf eine solche Haltung erwartet das Porträt Unverständnis bei der prototypischen Wählerschaft der LINKEN, das seien ostdeutsche Rentner und westdeutsche Arbeitslose. Ein Artikel aus 2015 nennt Kipping zumindest in Bezug auf die Themen Kriegsbeteiligung von Deutschland und Verteilung von Sozialleistungen im Vergleich zu Gysi als konsequent links.

Als Kippings herausragende Leistung wird nach dem Parteitag und nach der Bundestagswahl dann auch erwähnt, die konkurrierenden Flügel befriedet zu haben (2-mal).

Nach dem Spiegel steht Kipping inhaltlich für eine gewisse Neuausrichtung der Partei in eine moderate, entspannte Richtung, sowohl nach innen als auch nach außen. Andererseits bewertet das Magazin sie in ihren Positionen als noch nicht gefestigt.

\subsection{Themen}

Zur Beantwortung der Frage, in welchem Kontext sich die untersuchte Person womit beschäftigt, für die ich mich nun von den Formulierungen in den Texten entferne, wurden die Themen und Ereignisse gesammelt, mit denen der Name Kipping in den 45 Artikeln in Verbindung gebracht wird. Der dominierende Themenzusammenhang ist von Anfang an der Bundestagswahlkampf. Hier hinein sind Artikel zur Umverteilung der Steuerlast, Einführung der 30h-Woche, Deckelung von Managergehältern, Reformen bei Hartz IV und die Einführung ei-

16 Alles Beschreibungen aus dem Porträt. 
nes flächendeckenden Mindestlohns (3 Artikel) zu ordnen, die alle auf das Wahlprogramm der von Kipping vertretenen Partei verweisen. In enger Verbindung zu Landtags- und Bundestagswahlen stehen außerdem Artikel über Parteigenossen, genauer über Gregor Gysi (4 Artikel) und Sahra Wagenknecht (3 Artikel), außerdem Berichte über LINKEN-Parteitage (3 Artikel), in denen Wahlkampfprogramme bzw. -kandidaten sowie Gruppierungen innerhalb der Partei vorgestellt werden, dann Texte über andere Parteien, insbesondere die SPD, und schließlich für den Wahlkampf nutzbare Ereignisse wie Aktivitäten der BlockupyBewegung, die NSA-Affäre (4 Artikel) und den Krieg in Syrien, zu denen Kipping Stellung nimmt. Nach der Bundestagswahl werden in den Artikeln Reaktionen auf das Wahlergebnis, Kommentare zu den Koalitionsverhandlungen, wieder zu NSA (3 Artikel) sowie zur Diskussion um das Profil der Linkspartei veröffentlicht. Mehr oder weniger private Themen, die mit Kipping verbunden werden, sind der Tod des Parteigenossen Lothar Bisky sowie ein Bericht über einen falschen Priester.

Die Suche nach thematischen Häufungen, die für die Ermittlung von Standardwerten wichtig ist, führt zu einem Wahlkampf mit LINKEN-typischen Themen sowie Kippings wiederholtem Engagement bezüglich der NSA-Abhör-Affäre, die sich eignet, die alte und neue Bundesregierung zu kritisieren.

2015 werden Themen zur Verteilungspolitik und zu dem Verhältnis zur SPD weitergeführt. Größeren Raum nehmen Artikel zur Krise in Griechenland, zum Flüchtlingsstrom im Zusammenhang mit dem Syrienkrieg, zum Konflikt zwischen Russland und der Ukraine, aber auch zum Wechsel an der LINKEN-Fraktionsspitze sowie zu den Aktivitäten der BlockupyBewegung ein, die zeigen, dass Kipping als Sprecherin ihrer Partei fungiert.

\subsection{Aktivitäten und Sprachhandlungen}

Zur Erfassung der Kipping zugeschriebenen Aktivitäten und Sprachhandlungen wurde nach Prädikationen gesucht, die über die bereits bearbeiteten Themenfelder hinausweisen und Beschreibungen von in der jeweiligen konkreten Situation vollzogenen Handlungen inkl. Wünschen bzw. Aufgaben betreffen. Der Fokus der Analyse lag auf Verben und ihnen zugeordneten Adverbialen, aber auch Substantive mit Prädikatsfunktion wurden beachtet (ähnlich auch Ziem 2008b: 410). Diese habe ich nach semantisch verwandten Gruppen geordnet, so dass trotz sprachlicher Varianz inhaltliche Wiederholungen bzw. Wortfelder zu erkennen sind. Außerdem waren die Personen und Gruppen herauszuarbeiten, mit denen Kipping in Verbindung gebracht wird, um sie dann mit Kippings Handlungen in Relation zu setzen. Da das vorrangige Instrument politischen Handelns das gesprochene und geschriebene Wort ist, geben die gesammelten Einheiten zugleich gewisse Hinweise auf eingesetzte Mittel. Wie im Fragenkomplex 5 sticht das Porträt hier nicht als besonders hervor, weshalb dessen Formulierungen nicht gesondert markiert sind.

Unter den Einheiten, die keine bzw. nicht eindeutig Sprachhandlungen bezeichnen, fällt besonders das Wort- bzw. Metaphernfeld ,kämpfen/konkurrieren' auf, das variiert gefüllt ist: konkurrieren, sich einen Machtkampf liefern, einen Vorstoß machen, sich behaupten können, siegen, befrieden, noch nichts gewonnen haben, ein Wahlkampfteam zusammenstellen, öffent- 
licher Schlagabtausch, Duell, Überbietungswettkampf vermeiden. ${ }^{17}$ Kipping befindet sich nach Spiegel-Einschätzung offenbar in einem inner- und außerparteilichen Wettbewerb, innerparteilich mit Sahra Wagenknecht, außerparteilich mit anderen Parteien bzw. deren Vertretern, z. B. mit Andrea Nahles und Frank-Walter Steinmeier von der SPD, Angela Merkel von der CDU und mit der AfD. Das Wortfeld gibt es 2015 lediglich vereinzelt in der Beschreibung von Kippings Handlungen in Talkshows (keilen, gegeneinander antreten). Es ist offensichtlich typisch für die Wahlkampfberichterstattung und die von Talk-Shows, nicht aber für die Beschreibung jeglicher politischer Handlungen. Daneben habe ich eine Gruppe zu ,versuchen' gebildet: versuchen (2-mal), die Gelegenheit nutzen (2-mal), sich Mühe geben. Teile von Kippings Handlungen - nämlich ein Zentrum zu schaffen, eine Mindestlohninitiative in Gang zu bringen - werden als Versuche kategorisiert. Auch diese Gruppe ist 2015 fast nicht mehr vorhanden (sich bemühen, Schaden zu begrenzen). Nonverbales Verhalten wird beschrieben: lächeln, strahlen. In eine Mischgruppe sind nichts einfallen, der Versuchung widerstehen, vermeiden wollen, vorrechnen, wissen (2-mal), (Jeanne d'Arc) spielen, ködern subsumiert. Es fällt auf, dass nur wenige Beschreibungen körperlicher Tätigkeiten mit Kipping verbunden werden: kochen, betreten, rausfahren. ${ }^{18}$ Die Aufmerksamkeit des Spiegels liegt offensichtlich auf den sprachlichen Handlungen Kippings und dem Inhalt ihrer Aussagen, was einerseits an der vorrangig redewiedergebenden Berichterstattung liegt, andererseits an der typischen Handlungsform von PolitikerInnen - reden. Vielleicht verbirgt sich dahinter aber auch ein geschlechterrollenstereotypes Berichten, das körperliches Agieren von Frauen eher marginalisiert. ${ }^{19}$

Phrasen, mit denen Kippings sprachliches Verhalten beschrieben bzw. Zitate eingeleitet werden, sind ebenfalls gruppiert worden. Dabei sind für 2013 zwei Felder auffällig: a) Phrasen des Aufforderns: verlangen, auffordern (3-mal), zur Rückendeckung aufrufen, fordern (6mal), vorschlagen (2-mal), einen Vorschlag präsentieren und b) Phrasen des Kritisierens: vorwerfen (3-mal), scharf kritisieren, kritisieren, auszusetzen haben, drohen, scharf angreifen, als Heuchler bezeichnen, sich stören, zur Rede stellen, mit einer Spitze Ausdruck verleihen. Die größte Gruppe bilden redeeinleitende Wörter und Phrasen des Sagens i. w. S: c) sagen (20-mal), erklären (2-mal), entgegnen, meinen, betonen, schreiben, ins Mikrofon diktieren, mit potenziellen Wählern Persönliches besprechen, rufen, vorstellen, trotzig bekräftigen. d) Größere sprachliche Ereignisse werden erwähnt: Pressekonferenzen veranstalten, quietschvergnügt die Märchenstunde geben, eine Betroffenheitsrede halten. e) Ereignisse werden angekündigt: prognostizieren, ankündigen (3-mal). f) Schließlich könnte sich eine Gruppe als ,etwas durchsetzen (wollen)' sprachhandlerisch deuten lassen: sich stark machen, ein Versprechen abringen wollen.

Diese Sprachhandlungen, zum großen Teil bereits Interpretationen von Kippings verbalen Aktivitäten, werden zusätzlich, wenn auch nur zu einem geringen Maße kommentiert. Kip-

\footnotetext{
${ }^{17}$ In das Metaphernfeld des Kampfes gehören weiterhin die zu den Sprachhandlungen geordneten zur Rückendeckung aufrufen, scharf angreifen, mit einer Spitze Ausdruck verleihen. Es ist keine Häufung dieses Feldes im Porträt zu erkennen.

18 2015: treffen (4), entspannt zurücklehnen, zuhören, Hotel betreten, boykottieren. Es findet sich nichts zu Kippings nonverbalem Verhalten. Nicht eingeordnet wurden: nicht wechseln wollen, nicht fürchten.

${ }^{19}$ Das wäre in einem größeren Vergleichsprojekt zur Berichterstattung über verschiedenen Frauen, aber v. a. über Frauen und Männer in der Politik zu be- bzw. widerlegen.
} 
pings kommunikatives Auftreten deuten die Journalisten einerseits als scharf (2-mal), trotzig, mit einer Spitze, quietschvergnügt, leicht pikiert, andererseits als weitgehend sachlich (in Talkshow), aber auch ohne Idee. Daneben steht die generelle Einschätzung ihres Kommunikationsstils als (innerparteilich) moderierend, was als deutlich anders als Lafontaines Auftreten aufgefasst wird. Die genannten Adjektive deute ich wiederum als Hinweis darauf, dass die Autoren Kippings Sprachverhalten häufiger als emotional, ja sogar als kindlich beurteilen als sachlich.

Auffällig ist, wie Kippings Agieren gegenüber Angela Merkel und der Bundesregierung dargestellt wird. Die Formulierungen stammen vorrangig aus den Gruppen ,Fordern` und ,Kritisieren' und können als typisch für die Beschreibung oppositionellen Handelns gedeutet werden: fordern (5-mal), verlangen, auffordern, angreifen, einen Vorstoß machen, ködern, scharf kritisieren, Politikstil kritisieren, zur Rede stellen, vorwerfen (2-mal), drohen. Diese Formulierungen tragen dazu bei, dass die Regierung Merkel und die Bundeskanzlerin selbst noch vor DAX-Vorstand, Managern und Mitgliedern der früheren Regierung (Steinmeier) zu Gegnern Kippings aufgebaut werden. Weitere herausragende konkurrierende Mitspieler sind laut Spiegel wie erwähnt Wagenknecht, Lafontaine bzw. das Lafontaine-Lager. Hervorzuhebende Partner Kippings stellen Gysi und Kippings Ko-Parteivorsitzende Bernd Riexinger dar, ihr Wunschpartner sei die SPD. Schließlich spielt für Politiker die Wählerschaft eine wichtige Rolle. Diese sieht der Spiegel bei den LINKEN stereotyp in ostdeutschen Rentnern und westdeutschen Arbeitslosen, für Kipping dagegen eher bei rotweintrinkenden und alphabetisierten SchalträgerInnen. Kippings Zielgruppe und die der LINKEN stimmen offenbar nicht ganz überein. Nicht alle von Kippings „Mitspielern“ sind demnach typisch für eine LINKENPolitikerin. Allerdings sieht der Spiegel hier auch eine der wenigen formulierten Aufgaben bzw. Pflichten Kippings. Im Porträt findet sich nämlich eine Äußerung, die zu einer Aufgabe umgedeutet werden kann, das Finden neuer Wähler und Mitglieder: Sie muss noch beweisen, dass sie gegen den rasanten Mitgliederschwund und die Überalterung der Partei Mittel findet (Porträt 01.06.2013).

So eine Aufgabenformulierung findet sich in den Texten von 2015 nicht. Diese fokussieren vielmehr auf die Redewiedergabe Kippings zu jahresaktuellen Ereignissen, wozu keine Bundestagswahl gehörte. Dementsprechend ist die Anzahl der Phrasen aus der Gruppe Auffordern (fordern (2-mal), darauf drängen, mahnen) deutlich geringer, die Gruppe eher ersetzt durch Verben, die ich dem Befürworten zugeordnet habe: (nicht) wollen, plädieren, werben (2-mal), dafür sein, goldrichtig finden, feiern, an etwas festhalten, sich für etwas aussprechen, schwärmen (2-mal), den Rücken stärken. Es finden sich viele neutral redeeinleitende Wörter und Phrasen des Sagens (bezeichnen, sagen (6-mal), äußern, mitteilen, laut, nach Angaben, erklären (2-mal), Erklärung abgeben, verweisen auf, ausdrücken, zu Protokoll geben, (schreiben), begründen, Hauptrednerin sein, nacherzählen, ausrufen) und Meinens (meinen, sehen (2-mal), nach Ansicht, aus ihrer Sicht, nennen (3-mal)). Daneben gibt es auch die für oppositionelles Handeln typische Gruppe des Kritisierens/(Ver)urteilen: Entscheidung kritisieren, Kritikerin sein, verantwortlich machen, widersprechen, vorwerfen, sich distanzieren, dagegen halten, abstreiten, auf Distanz gehen, bedauern. Andere sprachliche Handlungen sind danken und einen Posten ablehnen, kleinreden und umdefinieren, letztere offensichtliche kritische Interpretationen bestimmter Äußerungen Kippings. Im Vergleich zum Jahr 2013 fallen die Beschreibungen von Kippings sprachlicher Performance also moderater und neutra-

ISSN 1615-3014 
ler aus. Der Spiegel interpretiert ihre Sprachhandlungen deutlich weniger wahlkämpferisch und auch nicht mehr als unsicher.

Kippings Gegner sind dann auch weniger häufig Merkel und die Regierung. Genannt werden auch die CSU, der Bundestag, die AfD, der Westen, Bundespräsident Gauck und die Polizei. Kippings Partner sieht der Spiegel 2015 vorrangig in ihrem Co-Parteivorsitzenden sowie der Blockupy-Bewegung. Eine Konkurrenz zu Wagenknecht kommt nicht mehr zur Sprache.

\section{$5 \quad$ Fazit und Ausblick}

Mit dem Zitat über die sich zu beweisende Parteivorsitzende ist ein Beispiel gegeben, dass auf eine Prädikation hinweist, welche sich in den verschiedenen Spiegel-Slots zur Person Kipping in unterschiedlicher Form wiederfindet. Fasst man die sich, wenn auch nicht häufig, aber dennoch wiederholenden Beschreibungen für 2013 zusammen, ergibt sich ein Bild einer jungen, selbstbewussten, für eine LINKE nicht ganz typischen, für viele auch noch unbekannten und damit noch nicht etablierten, weiblichen Parteivorsitzenden, die die Flügel ihrer Partei durch ihren moderaten Ton mit der Zeit zwar befriedet hat und die Regierung in und nach dem Bundestagswahlkampf 2013 oppositionstypisch herausfordert, die aber trotz ihrer bisherigen offensichtlichen, aber kaum genannten Karriereerfolge - sie ist immerhin Co-Vorsitzende einer Partei - ideologisch und in ihrer Position als nicht gefestigt eingeschätzt wird und sich insbesondere gegenüber ihren KonkurrentInnen aus den eigenen Reihen zu beweisen hat. Zu diesem Bild trägt insbesondere das Porträt Süße Katja gegen rote Sahra (06.01.2013) bei, in dem Kipping zusammenfassend mit Jeanne d'Arc verglichen wird. Da es jedoch kaum zu einer Wiederholung eines solchen Textes in Spiegel bzw. SPON kommt, muss das gezeichnete Bild als blass oder wie die untersuchte Person vom Medium selbst als noch nicht etabliert eingestuft werden. Die Aussage, dass man Kipping vielleicht unterschätze (06.01.2013), und sie zudem als nicht festgelegt beurteilt, kann ebenfalls daraufhin gedeutet werden, dass der Spiegel selbst noch kein festes und schon gar kein differenziertes Bild von Kipping hat. Auch wenn die anderen 44 Texte an Kipping kaum anders als als Sprachrohr ihrer Partei interessiert sind $^{20}$, was sich noch mehr in den Artikeln aus dem Jahr 2015 zeigt und ich auf ihren Status als Chefin, jedoch nicht Fraktionsvorsitzende einer nicht sehr einflussreichen und sogar umstrittenen Oppositionspartei zurückführen möchte, stützen sie das gezeichnete Bild in Bezug auf den Bekanntheitsgrad, das oppositionstypische kritisierende und fordernde Sprachhandeln, das von ihr kommunizierte LINKEN-typische Wahlprogramm und den moderaten innerparteilichen Ansatz Kippings. Bemerkenswert, aber dennoch dazu passend ist die mehrfache Bewertung des sprachlichen Handelns Kippings als trotzig, pikiert, quietschvergnügt 2015 auch schwärmend - was ich als emotional und kindlich zusammengefasst habe und ihr Image in Spiegel und SPON damit abschließend und zugespitzt als das Mädchen an der Spitze der Opposition beschreiben möchte.

Diese Beschreibung habe ich mithilfe eines an dem Frame-Slot zur Person in berufsbezogener Rolle (cf. v. a. Ziem 2008: 416-418) orientierten, aber in Fragenbündeln zusammengefassten und zudem leicht erweiterten Fragenkatalog herausgearbeitet. Das ist geschehen, um dem Frame Person im politischen Berufsfeld besser gerecht zu werden und nicht zuletzt auch die

${ }^{20}$ Also weder an ihr als Privatperson noch an ihrem politischen oder privaten Lebenslauf.

ISSN 1615-3014 
Beschreibung und Bewertung der sprachlichen Performance, welche in der Politik eine große Rolle spielt, erfassen zu können. Die Untersuchung diente auch dazu, diese Fragenbündel als Grundlage für Image- bzw. Stereotypenanalysen zu PolitikerInnen auszuprobieren. Dabei erscheint mir die Gruppe 6 zu den Aktivitäten im Nachhinein als zu komplex. Zu überlegen ist, ob die „Mitspieler“ in einen eigenen Punkt ausgelagert und ihre Aktivitäten außerdem besser bzw. überhaupt erfasst werden sollten, da PolitikerInnen konstant unter Beobachtung stehen, von verschiedenen Interessengruppen beeinflusst werden und zudem KonkurrentInnen und Gegner, aber auch Verbündete besitzen, die sich der Person gegenüber auf eine bestimmte Art verhalten. So kann man die Bedingungen, unter denen eine Person agiert, und damit die Reaktionen auf das Verhalten der untersuchten Person besser erfassen. Auch wenn im Falle Kippings der private Hintergrund nicht sehr stark ausgeleuchtet war, so denke ich weiterhin, dass die Berichte über die privaten Verhältnisse von PolitikerInnen für die Auffassung bzw. das Bild, das Wähler sich über sie machen, von Bedeutung sind.

Inhaltlich scheint mir für kommende Untersuchungen die Berücksichtigung von folgenden Fragen zum Image von Politikerinnen interessant, die sich hier andeuteten, aber aufgrund der Fokussierung auf eine Person nicht beantwortet werden konnten: 1) Wie wird neben dem verbalen und paraverbalen das nonverbale Verhalten von PolitikerInnen beschrieben und bewertet? Trägt es zu einer geschlechterrollenstereotypen Berichterstattung bei? Strahlen und lächeln noch andere Frauen als Kipping in den Medien oder auch Männer? 2) Bei Kipping spielte, wenn auch nicht häufig, doch auf unterschiedliche Weise ihr Alter eine Rolle, was in meiner Deutung des Spiegel-Bildes in den Begriff des Mädchens mündete. Interessant wäre es herauszuarbeiten, ob bzw. wie neben Geschlechterrollenstereotypen auch Altersstereotypen in der Berichterstattung wirken. Den typischen Politiker stellt man sich wohl eher als alten und hoffentlich - weisen Mann denn als junge, frisch von der Universität kommende Frau vor. Hier wäre auch ein Vergleich mit anderen Ländern wie zum Beispiel Dänemark, wo gegenwärtig (2015) viele junge Frauen in der Regierung sitzen, ergiebig, um zu schauen, wie über Frauen im selben Alter, aber in verschiedenen Gesellschaften berichtet wird.

\section{Literatur}

Burger, Harald/Luginbühl, Martin (2014): Mediensprache. Eine Einführung in Sprache und Kommunikationsformen der Massenmedien. Berlin/Boston: de Gruyter.

Busse, Dietrich (2012): Frame-Semantik. Ein Kompendium. Berlin/Boston: de Gruyter.

DDB $=$ Der deutsche Bundestag (s. a.): Abgeordnete in Zahlen. www.bundestag.de/bundestag /abgeordnete18/mdb_zahlen/frauen_maenner/260128 [18.04.2015].

Der Spiegel (2013, 2015). Hamburg: Spiegel.

Drinkmann, Nancy/Cabllero, Claudio (2007): „Eine Frau ist eine Frau ist eine Frau? Die Berichterstattung über die Kandidaten der Bundespräsidentenwahl 2004“. In: Holtz-Bacha, Christina/König-Reiling, Nina (eds.): Warum nicht gleich? Wie die Medien mit Frauen in der Politik umgehen. Wiesbaden, VS Verlag für Sozialwissenschaften: 167-203.

Fraas, Claudia (1996): Gebrauchswandel und Bedeutungsvarianz in Textnetzen: die Konzepte „Identität“ und „Deutsche“ im Diskurs zur deutschen Einheit. Tübingen: Narr.

Girnth, Heiko (2002): Sprache und Sprachverwendung in der Politik. Tübingen: Niemeyer. 
Heier, Anke (2016): „Dänischer Rechtsextremismus und Tyskertøser - Zu nationalen Stereotypen in ein- und zweisprachigen Wörterbüchern“. In: Hallsteinsdóttir, Erla et al. (eds.): Perspektiven der Stereotypenforschung. Frankfurt a. M., Lang: 13-34.

Holtz-Bacha, Christina (2007a): „Mit den Waffen einer Frau? Politikerinnen im Wahlkampf“. In: Holtz-Bacha, Christina/König-Reiling, Nina (eds.): Warum nicht gleich? Wie die Medien mit Frauen in der Politik umgehen. Wiesbaden, VS Verlag für Sozialwissenschaften: 79-104.

Holtz-Bacha, Christina (2007b): „Zur Einführung: Politikerinnen in den Medien“. In: HoltzBacha, Christina/König-Reiling, Nina (eds.): Warum nicht gleich? Wie die Medien mit Frauen in der Politik umgehen. Wiesbaden, VS Verlag für Sozialwissenschaften: 7-16.

Klein, Josef (1998): „Linguistische Stereotypenbegriffe. Sozialpsychologischer vs. semantiktheoretischer Traditionsstrang und einige frametheoretische Überlegungen“. In: Heinemann, Margit (ed.): Sprachliche und soziale Stereotype. Frankfurt a. M., Lang: 25-46.

Konerding, Klaus-Peter (1993): Frames und lexikalisches Bedeutungswissen: Untersuchungen zur linguistischen Grundlegung einer Frametheorie und zu ihrer Anwendung in der Lexikographie. Tübingen: Niemeyer.

Konerding, Klaus-Peter (2001): „Sprache im Alltag und kognitive Linguistik: Stereotype und schematisiertes Wissen“. In: Lehr, Andrea et al. (eds.): Sprache im Alltag. Beiträge zu neuen Perspektiven in der Linguistik. Berlin/New York, de Gruyter: 151-172.

Lippmann, Walter (1922): Public Opinion. New York: Macmillan.

Maurer, Marcus/Reinemann, Carsten (2006): Medieninhalte. Eine Einführung. Wiesbaden: VS Verlag für Sozialwissenschaften.

Meyer, Birgit (2009): „Nachts, wenn der Generalsekretär weint - Politikerinnen in der Presse“. APuZ 50: 9-15.

Niehr, Thomas (2014): Einführung in die Politolinguistik. Göttingen: Vandenhoeck \& Ruprecht.

Quasthoff, Uta (1998): „Stereotype in Alltagsargumentationen: Ein Beitrag zur Dynamisierung der Stereotypenforschung“. In: Heinemann, Margot (ed.): Sprachliche und soziale Stereotype. Frankfurt a. M., Lang: 47-72.

Schröter, Melanie/Carius, Björn (2009): Vom politischen Gebrauch der Sprache. Wort, Text, Diskurs. Eine Einführung. Frankfurt a. M.: Lang.

SPON = Spiegel online $(2013,2015)$. www.spiegel.de [10.02.2016].

STATISTA = Das Statistik-Portal. http://de.statista.com/statistik/daten/studie/13232/umfrage /auflage-der-wochenzeitschrift-der-spiegel-seit1995/ [21.05.2016].

Van Dijk, Teun A. (1984): Prejudice in Discourse. Amsterdam/Philadelphia: Benjamins.

Veith, Werner H. (2005): Soziolinguistik. Ein Arbeitsbuch mit Kontrollfragen und Antworten. 2. Auflage. Tübingen: Narr.

Wengeler, Martin (2012): „Der Angriff auf das Bestehende. Zur Erinnerungskultur an ,1968“ in deutschen Printmedien am Beispiel des SPIEGEL“. In: Heidrun Kämper/Joachim Scharloth/Martin Wengeler (eds.): „1968“. Eine sprachwissenschaftliche Zwischenbilanz. Berlin/New York, de Gruyter: 55-81.

Ziem, Alexander (2008a): „Frame-Semantik und Diskursanalyse - Skizze einer kognitionswissenschaftlich inspirierten Methode zur Analyse gesellschaftlichen Wissens“. In: Warn- 
ke, Ingo H./Spitzmüller, Jürgen (eds.): Methoden der Diskurslinguistik. Sprachwissenschaftiche Zugänge zur transtextuellen Ebene. Berlin/New York, de Gruyter: 89-116.

Ziem, Alexander (2008b): Frames und sprachliches Wissen. Berlin/New York: de Gruyter. 